\title{
Neutrophilic eccrine hidradenitis (NEH) caused by letrozole: A Case Report
}

\section{Ameen Alawadhi MD, Jawaher Aljalahma MD, Khalid Bin Thani MD*}

Salmaniya Medical Complex, Manama, Kingdom of Bahrain

*Corresponding Author: Khalid Bin Thani MD, Salmaniya Medical Complex, Manama, Kingdom of Bahrain

Received date: 09 August 2021; Accepted date: 17 August 2021; Published date: 21 August 2021

Citation: Alawadhi A, Aljalahma J, Thani KB (2021) Neutrophilic eccrine hidradenitis (NEH) caused by letrozole: A Case Report. J Med Case

Rep Case Series 2(8): https://doi.org/10.38207/JMCRCS/2021/0208124

Copyright: (C) 2021 Khalid Bin Thani MD. This is an open-access article distributed under the terms of the Creative Commons Attribution

License, which permits unrestricted use, distribution, and reproduction in any medium, provided the original author and source are credited.

\section{Introduction}

Chemotherapy can lead to cutaneous manifestations ranging from an adverse reaction to infectious complications caused by immunosuppression and neutropenia [1].

Unfortunately, it is usually misdiagnosed as hypersensitivity reaction, contact dermatitis, graft versus host disease, or vasculitis. Definitive diagnosis is important to avoid unnecessary, potentially harmful therapeutic intervention in the future treatments plan. In 2008, Toxic erythema of chemotherapy (TEC) was introduced as a new clinically descriptive term. The range of entities classified under TEC is broad and includes acral erythema (palmar-plantar erythrodysesthesia, hand-foot syndrome), the intertriginous eruption of chemotherapy, and neutrophilic eccrine hidradenitis (NEH). Therefore, in this case report, we discuss a rare presentation of $\mathrm{NEH}$ related to letrozole usage.

\section{Case Report}

A 58-year-old female is known to have diabetes mellitus, hypothyroidism, diabetic peripheral neuropathy, and left breast cancer. Her malignancy history started in 2014 when she was found to have a left breast mass. Following ultrasonography, mammography, and core biopsy the lump was diagnosed to be infiltrative ductal carcinoma. Accordingly, wide local excision of the
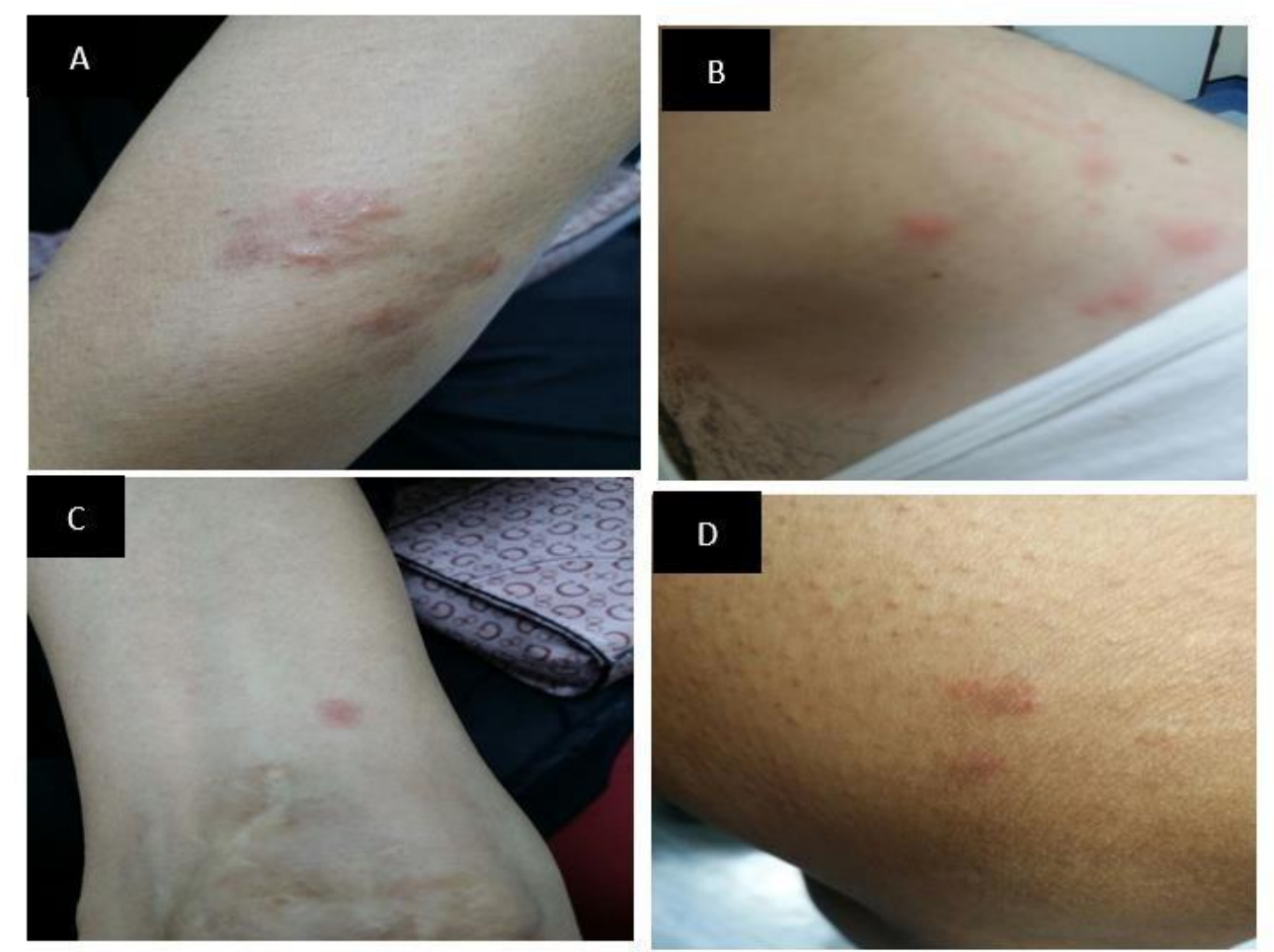

Figure 1: The upper and lower extremities revealing erythematous and edematous painful pruritic papules

left breast mass was done with axillary lymph dissection and the sample was sent for histopathological examination. The histopathological examination confirmed the diagnosis of invasive ductal carcinoma grade 1, stage T1N1M0. Immunohistochemistry Studies showed Estrogen Receptor (ER) positive, Progesterone Receptor (PR) positive, Her2 \new was also positive. The line of management decided for this lady included external beam radiotherapy (EBRT), with 6 cycles of chemotherapy including cyclophosphamide, trastuzumab, and tamoxifen as based hormonal therapy. The patient kept on tamoxifen for 3 years. In 2017, the tamoxifen was changed to letrozole $2.5 \mathrm{mg}$ daily. Hence, the patient presented with a red pimple in the neck and lower extremities after 3 days of taking letrozole. The lesions were itchy. She did not report fever. There was no history of any neurological, respiratory, cardiac, urinary, or psychiatric symptoms, nor did she have any gastrointestinal symptoms. Also, the patient denied taking any new medication except for letrozole. On examination, there were multiple erythematous papules involving the posterior part of the neck and lower extremities (Figure 1). No lymphadenopathy was appreciated and other systems' examinations were unremarkable. Neutrophilic Eccrine Hidradenitis (NEH) was on the top of our differentials and skin biopsy confirmed the diagnosis (Figure 2). 


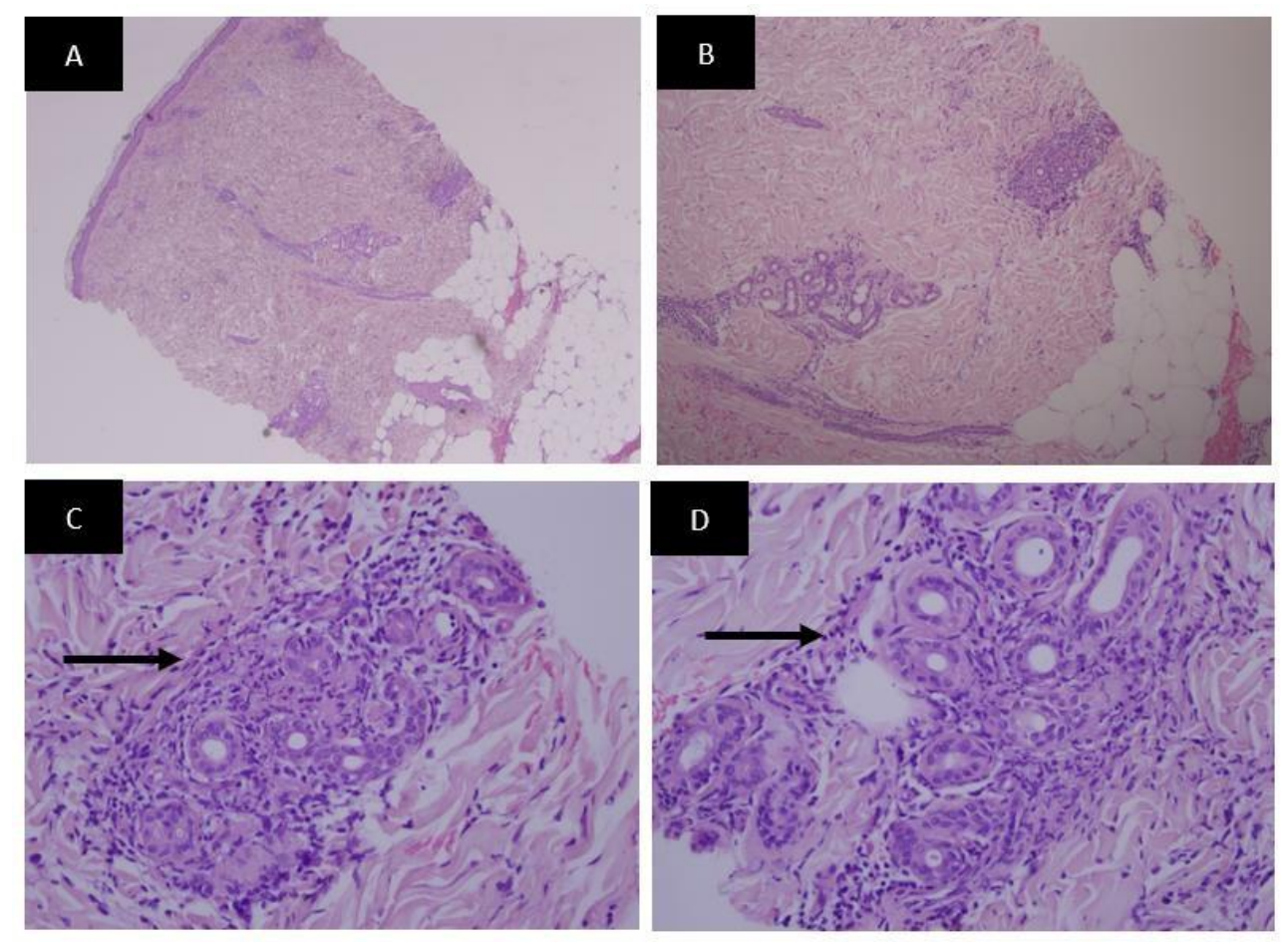

Figure 2: The skin biopsy histopathology slides - The skin sections show the dermis with Neutrophilic Infiltrate around eccrine glands (arrows) and into the walls of deep dermal blood vessels. There is also superficial and deep perivascular lymphocytic infiltrate. No leukocytoclastic vasculitis is seen. No dysplasia or malignancy is seen.

\section{Discussion}

Neutrophilic Eccrine Hydraneitis (NEH) is a rare reactive disorder. It was first described by Harrist et al. in 1982 [2] and most reported cases have been observed in the setting of acute myelogenous leukemia [3-4]. It was also recognized in association with infections, such as human immunodeficiency virus (HIV), bacterial staphylococcus and streptococcus infections, certain medications, hemodialysis, and even in healthy individual [5-10]. Lesions can appear anywhere from 2 days to 2 years after initiation of chemotherapy. More than $70 \%$ of oncology patients who develop neutrophilic eccrine hidradenitis do so after their first course of chemotherapy [10]. The known drugs reported inducing NEH were bleomycin, chlorambucil, cyclophosphamide, cytarabine, doxorubicin, lomustine, mitoxantrone, topotecan, and vincristine [11]. The most common of these is cytrabine [12] and as far as we know this is the first reported case of NEH induced by letrozole.

The pathogenesis of NEH is unclear. Two major hypotheses have been proposed. First, NEH may be the consequence of a direct toxic effect of a drug secreted in the sweat from eccrine coils and duct cells. Cases of neutrophilic eccrine hidradenitis resolving after withdrawal of chemotherapy and recurring upon reinstitution of the same regimen favor the former. Also, supporting a direct toxic drug response is a study showing that the intradermal injection of bleomycin can yield local neutrophilic eccrine hidradenitis changes [13]. In contrast, other researchers have proposed that NEH is a hypersensitivity reaction within the spectrum of neutrophilic dermatosis such as sweet syndrome. NEH in patients with no previous treatment supports this hypothesis.
NEH is an infrequent disease. The only study done was by Bachmeyer \& Aractingi in 1982 and 1998, who found only 51 cases. Most of these cases were ponded to leukemia. A slight male predominance ${ }^{14}$ and has been reported in individuals as young as 6 months and as old as 79 years.

Patients with NEH develop skin lesions and frequently report fever. It is characterized by erythematous and edematous plaques or papules of variable size that may be purpuric and painful. They are usually located on the extremities, trunk, face, and palms. Histologically, $\mathrm{NEH}$ demonstrates neutrophilic infiltrate selectively distributed around the eccrine glands and coils and is associated with necrosis. NEH is typically a self-limited process. The treatment options include non-steroidal anti-inflammatory drugs (NSAIDs), steroids as well as dapsone. Fortunately, it does not appear to portend a worse prognosis for the underlying malignancy when occurring in that setting.

\section{Conclusion}

Neutrophilic eccrine hidradenitis (NEH) is an uncommon disease frequently seen in the setting of malignancy or patients receiving chemotherapy as in our patient. In the available literature, we found some reported cases regarding skin lesions induced by letrozoles such as letrozole-induced eczematous skin eruption [14] and letrozoleinduced leukocytoclastic small vessel vasculitis [15]. As far as we know, this is the first reported case of letrozole induced neutrophilic eccrine hidradenitis (NEH). It is important to keep in mind such possible skin side effects of letrozole, particularly because it is a commonly used drug in breast cancer treatment.

Conflict of interest: None Declared 


\section{References}

1. Bolognia JL, Cooper DL, Glusac EJ (2008) Toxic erythema of chemotherapy: a useful clinical term. J Am Acad Dermatol. 59(3): 524-9.

2. Harrist TJ, Fine JD, Berman RS, Murphy GF, Mihm MC Jr (1982) Neutrophilic eccrine hidradenitis: a distinctive type of neutrophilic dermatosis associated with myelo genous leukemia and chemotherapy. Arch Dermatol. 118(4): 263-266.

3. Fitzpatrick JE, Bennion SD, Reed OM, Wilson T, Reddy VV, et al. (1987) Neutrophilic eccrine hidradenitis associated wiih induction chemotherapy. J Cutan Pathol. 14(5): 272-278.

4. Allégué F. Soria C, Rocamora A, Muñoz E, Freire-Murgueytio P, et al. (1990) Neutrophilic eccrine hidradenitis in two neutropenic patients. J Am Acad Dermatol. 22(6 pt 1): 1110-1113.

5. Manganoni AM, Facchetti F, Gavazzoni R, Marocolo D, De Panfilis G (1994) Neutrophilic eccrine hidradenitis in a healthy woman. Dermatology. 189(2): 211-212.

6. Mercader-García P, Vilata-Corell JJ, Pardo-Sanchez J, ForteaBaixauli JM (2003) Neutrophilic eccrine hidradenitis in a patient with Behçet's disease. Acta Derm Venereol. 83(5): 395-396.

7. Bachmeyer C, Reygagne P, Aractingi S (2000) Recurrent neutrophilic eccrine hidradenitis in an HIV-1-infected patient. Dermatology. 200(4): 328-330.
8. Shih IH, Huang YH, Yang CH, Yang LC, Hong SH (2005) Childhood neutrophilic eccrine hidradenitis: a clinicopathologic and immunohistochemical study of 10 patients. J Am Acad Dermatol. 52(6): 963-6.

9. Takai T, Matsunaga A (2006) A case of neutrophilic eccrine hidradenitis associated with streptococcal infectious endocarditis. Dermatology. 212(2): 203-205.

10. Cohen PR (2007) Neutrophilic dermatoses occurring in oncology patients. Int J Dermatol. 46(1):106-11.

11. Susser WS, Whitaker-Worth DL, Grant-Kels JM (1999) Mucocutaneous reaction to chemotherapy. J Am Acad Dermatol. 40(3): 367-98.

12. Bachmeyer C, Aractingi S. Neutrophilic eccrine hidradenitis. Clin Dermatol 2000; 18: 315-318.

13. Bachmeyer C, Aractingi S (2000) Neutrophilic eccrine hidradenitis. Clin Dermatol. 18(3): 319-330.

14. Tripathy A, Kumari KM, Mohan Babu AV, Pai SB, Kumar D, M (2014) Anything rare is possible: letrozole induced eczematous skin eruption. J Clin Diagn Res. 8(6): YD03-YD04.

15. Pathmarajah P, Shah K, Taghipour K, Ramachandra S, Thorat MA, et al. (2015) Latrozole induced leukocytoclastic small vessel vasculitis. Int J Surg Case Rep. 16: 77-80. 